\title{
Transient Thermal Elastohydrodynamic modeling of cam-follower systems: understanding performance
}

\author{
Jonathan Raisin ${ }^{1,2}$, Nicolas Fillot ${ }^{2}$, Philippe Vergne ${ }^{2}$, David Dureisseix ${ }^{2}$, Vincent Lacour ${ }^{1}$ \\ ${ }^{1}$ Centre de Recherche de Solaize (CReS), Total Marketing \& Services, Solaize, France \\ 2 Université de Lyon, INSA-Lyon, CNRS, LaMCoS, UMR5259, F-69621, Villeurbanne, France
}

DOI: 10.1080/10402004.2015.1110865

\begin{abstract}
This article deals with the lubrication of cam-follower contacts in automobile racing applications. Timedependent Thermal Elastohydrodynamic line contact simulations are performed to analyze the contact performance achieved with a shear-thinning lubricant under highly dynamic conditions. Comparisons between different simulations are used to quantify the respective influence of shear-thinning, thermal softening and transient effects on friction and film thickness. Furthermore, this article highlights the formation and transport of a transient dimple responsible for an increase in lift close to the conditions where reversals of entrainment occur. Temperature distributions across the film thickness and pressure variations are reported to discuss the underlying phenomenon.
\end{abstract}

\section{INTRODUCTION}

Internal combustion engines (ICEs) in modern racing cars are some of the most highly stressed pieces of machinery on the planet. With rotational speeds possibly exceeding ten thousand revolutions per minute, the physical stresses to which the moving mechanical components of the engine are subjected are enormous. In the valve train system, for instance, the lubricated cam-follower contacts undergo dramatic changes of speed and load in a matter of a few milliseconds. Because reliability and high performance of each subsystem of the engine are both essential to win races, the search for an optimal combination of machinery and lubrication is relentless. For the mechanical components, the design strategy usually consists in using high-grade materials for their strength and stiffness along with precise calculation methods to optimize stress distribution and keep the moving mass as low as possible. For lubrication, the primary objective is twofold: the lubricant must not only create a protective film thick enough to prevent or limit the direct contact between the metallic surfaces, which would lead to wear and potential failure, but also minimize friction to recover the largest possible amount of the energy released during combustion (Total Competition (1)). In both cases, the lubricant viscosity is often the key parameter. Put simply, the optimal lubricant viscosity should be sufficiently high in the inlet of the contact so that the produced hydrodynamic lift can fully separate the solid surfaces and should remain as low as possible in the central/pressurized region to reduce friction due to the shearing of the fluid.

In order to fulfill this requirement, engine designers and lubricant manufacturers can exploit two different competing and often coexisting phenomena in lubrication: shear-thinning and thermal 
softening. Shear-thinning refers to the ability of some fluids, usually containing polymers, to exhibit a drop in viscosity when subjected to shear stress exceeding a certain threshold (Bair (2), Mary et al. (3)). By adjusting this threshold and the intensity of the drop with regards to the actual stresses experienced by the lubricant in a contact, the fluid friction can theoretically be significantly reduced with limited consequences on the film thickness. Thermal softening denotes the decrease of a fluid viscosity induced by a temperature rise. In some contacts found in the ICEs of racing cars, the extreme prevailing conditions of pressure, velocity and sliding produce tremendous amount of heat through the compression and the shearing of the lubricant. Therefore, any technical solution able to increase temperature locally (but below the point where lubricant degradation occurs) by disrupting the heat removal, such as the introduction of thermally insulating surface coatings (Björling et al (4), Habchi (5)), can also lead to significantly reduced fluid friction with limited consequence on the film thickness. In theory, taking advantage of one or both of these phenomena would allow to develop efficient lubricant for automobile racing engine applications. However, such a tuning requires an accurate knowledge of the actual conditions of pressure, shear and temperature within the film of lubricant which is hardly accessible experimentally, even with state-of-the-art apparatus (Ciuli et al. (6)).

From the early analyses relying on semi-analytical formulae (Dowson et al. (7), Bedewi et al (8)) to the more recent CFD based models (Chong et al. (9), Zavos and Nikolakopoulos (10)), the numerical analysis of the lubricated subsystems of the ICEs has greatly progressed. For instance, the use of transient EHL simulations (Ai and Yu (11), Messe and Lubrecht (12), Wang and Yang (13)) has played a major role in the understanding of the lubrication of cam-follower contacts upon which this article focuses. Among notable contributions, the work of Wang and Yang (Wang and Yang (13)) highlighted the importance of thermal effects on the performance of these specific contacts and, in particular, the influence of the "temperature-viscosity wedge mechanism" at work during zero entrainment velocity (ZEV) conditions. With the recent developments in the transient Thermal EHL modeling of contacts (Raisin et al. (14)) and in the high-pressure methods for the rheological characterization of lubricants (Bair (2), Vergne and Bair (15)), it now becomes possible to further explore the physical phenomena prevailing within the lubricant film. In this regard, the present study uses numerical simulation to investigate the respective contribution of shear-thinning, thermal softening and transient effects to the performance (film thickness and friction) of a lubricated cam-follower contact of an actual racing car. In addition, the build-up of a dimple and its transport throughout the contact during the ZEV conditions is exemplified. Comparisons between thermal and isothermal results are used to discuss the influence of both the "(transient) squeeze" and the "temperature-viscosity wedge" mechanisms and to assess the dominant one.

\section{CONTACT PROPERTIES}

\subsection{Operating conditions}

This article focuses on the transient lubricated line contact (Figure 1) formed between one of the cam and follower pairs found in the valve train system of an ICE.

The application of interest being automobile racing, highly dynamic variations of load, $w(t)$, entrainment velocity, $u_{m}(t)=\frac{1}{2}\left(u_{1}(t)+u_{2}(t)\right)$, and surface curvatures, $R_{1}(t)$ and $R_{2}(t)$, were retained to match the typical operating conditions of their ICEs. Although the exact profiles corresponding to these inputs are not reported herein for confidentiality issues, dynamical variations of the contact parameters are represented in Figure 2 through the variation of the classical Moes dimensionless parameters (Moes (16)) and through the variation of the lubricant transit time $t_{h}$. Moes 
dimensionless parameters, $\mathrm{M}$ and $\mathrm{L}$, can provide a fairly accurate approximation (assuming a stationary isothermal Newtonian lubrication regime) of the minimum film thickness in the contact for the most part of the cycle. They however are of limited use to represent the transition through ZEV conditions where both transient and thermal effects help maintaining a non-zero film thickness. The characteristic time, $t_{h}$, here approximated as the ratio of the instantaneous Hertzian half-width, $b(t)$, and entrainment velocity, $u_{m}(t)$, measures how fast lubricant is driven from the contact inlet to its center (Raisin et al. (14)). Over a cycle, consisting in the full rotation of the camshaft, $t_{h}$ remain mostly below one tenth of a millisecond, providing an idea of the extreme level of dynamics prevailing in the contact. Note that at the considered engine rotational speed, the cycle lasts about $6.67 \mathrm{~ms}$.

\subsection{Materials}

From a material point of view, the cam and follower are made of alloy steel, the properties of which are detailed in Table 1: $\rho$ is the density, $E$ is Young's Modulus, $v$ is Poisson's ratio, $c$ is the specific heat capacity and $k$ is the thermal conductivity. Contacting surfaces of both components are considered smooth to represent the superfinishing of real surfaces. Finally, the contact environment is regulated to a temperature $T_{0}=110^{\circ} \mathrm{C}$ by a constant supply of fresh lubricant.

The lubricant under consideration is rather typical of automotive applications. It is a blend composed of a hydrocracked mineral base oil, from group III (API classification) and PISH polymers with a concentration of $1.2 \%$ in weight (Mary et al. (3)). Thermophysical properties of the blend were extensively characterized and modeled by Mary et al. (Mary et al. (3), (17)) over significant ranges of pressure, temperature and shear stress. For more details the reader is referred to these papers. The temperature-modified Tait equation of state was chosen to represent the variations of the lubricant density with pressure and temperature:

$$
\begin{aligned}
\rho(p, T)= & \rho_{r} \frac{V_{r}}{V_{0}} \times \frac{V_{0}}{V} \\
\text { with } \quad \frac{V_{0}}{V} & =1-\frac{1}{1+K_{0}^{\prime}} \ln \left[1+\frac{p}{K_{0}}\left(1+K_{0}^{\prime}\right)\right] \\
& \frac{V_{r}}{V_{0}}=1+a_{v}\left(T-T_{r}\right) \\
K_{0} & =K_{00} \exp \left(-\beta_{K} T\right)
\end{aligned}
$$

where $V$ is the total volume at the temperature $T$ and pressure $p, V_{0}$ and $K_{0}$ the total volume and bulk modulus at temperature $T$ and atmospheric pressure $p_{0}$, respectively and $a_{v}$ the thermal expansivity. In practice, $K_{00}=9 \mathrm{GPa}$ and $\beta_{K}=6.1810^{-3} \mathrm{~K}^{-1}$ provide accurate values of $K_{0}$ for a large range of temperatures.

The modified WLF-Yasutomi correlation (Bair et al. (18)) defines the Newtonian (or low shear) viscosity of the fluid, $\eta_{1}(p, T)$, as:

$$
\begin{gathered}
\eta_{1}(p, T)=\eta_{g} \times 10^{\frac{-C_{1}\left(T-T_{g}(p)\right) F_{m}(p)}{C_{2}+\left(T-T_{g}(p)\right) F_{m}(p)}} \\
\text { with } \quad T_{g}(p)=T_{g}\left(p_{0}\right)+A_{1} \ln \left(1+A_{2} \times p\right) \\
F_{m}(p)=\left(1+B_{1} \times p\right)^{B_{2}}
\end{gathered}
$$


where $\eta_{g}$ is the viscosity at the glass transition temperature, $T_{g}(p)$, and $F_{m}(p)$ represents the dimensionless relative thermal expansion of the free volume. $T_{g}\left(p_{0}\right)$ is the glass transition of the considered fluid at atmospheric pressure and $A_{1}, A_{2}, B_{1}, B_{2}, C_{1}$ and $C_{2}$ are additional fluid-specific parameters.

The generalized Newtonian viscosity, $\eta(p, T, \tau)$ is then obtained from the modified Carreau-Yasuda formula (Bair (2)):

$$
\eta(p, T, \tau)=\eta_{1}(p, T)\left(\eta_{2} / \eta_{1}+\frac{1-\eta_{2} / \eta_{1}}{\left[1+\left(\frac{\tau}{G}\right)^{a}\right]^{\left(\frac{1}{n}-1\right) / a}}\right)
$$

where $\eta_{1}(p, T)$ is the low shear viscosity given by eq. (2), $\eta_{2} / \eta_{1}$ the relative viscosity of the second Newtonian plateau, $n$ the power law exponent, $a$ a parameter mainly impacting the slope of the viscosity vs. shear-stress curve, $\tau$ the shear stress, and $G$ the effective shear modulus.

All the fluid related properties, including the parameters involved in the three models, are summarized in Table 2.

\subsection{Characteristic numbers}

Different characteristic numbers of interest in lubrication (Habchi et al. (19)), used for analysis purposes, are recalled in this section. These characteristic numbers are the slide-to-roll ratio (SRR), the Nahme-Griffith number ( $\mathrm{Na}$ ), the Weissenberg number (Wi) and the Limiting Shear Stress number (Li). It is important to note at this point that unlike for the SRR, the value of which can straightforwardly be obtained from the contact kinematics, the calculation of $\mathrm{Na}$, Wi and Li requires the accurate knowledge of the conditions prevailing within the contact. Numerical values used in this article for these three numbers were therefore computed directly from simulation results at the contact center, using averaged values across the lubricant film thickness for the viscosity, pressure, temperature, shear rate and shear stress.

The Slide-to-Roll Ratio, SRR, characterizes the amount of sliding experienced by a given contact. It is defined as the ratio of the sliding velocity, $u_{s}=u_{2}-u_{1}$, and the entrainment velocity, $u_{m}$ :

$$
\mathrm{SRR}=2 \frac{u_{2}-u_{1}}{u_{1}+u_{2}}
$$

The Nahme-Griffith number, $\mathrm{Na}$, is a measure of how much viscous dissipation affects the temperature dependent viscosity (Winter (20)). The threshold value of $\mathrm{Na}$ above which thermal effects may not be disregarded seems to depend on the nature of the considered fluid (Bair (2), Winter (20)). Nonetheless, a value close to or above unity is a good indicator of the presence of significant thermal softening. Following Winter's work, Na reads (Winter (20)):

$$
\mathrm{Na}=\frac{\beta \eta\left(p, T_{0}, \tau\right) \dot{\gamma}^{2} h_{c}^{2}}{k}
$$

where $\beta=-\partial \ln \eta(p, T, \tau) / \partial T$ is the temperature-viscosity coefficient, $\dot{\gamma}$ the shear rate, $h_{c}$ the film thickness and $k$ the lubricant thermal conductivity. $\eta\left(p, T_{0}, \tau\right)$ is the generalized Newtonian viscosity at pressure $p$, shear stress $\tau$ and ambient temperature $T_{0}$.

The Weissenberg number, $\mathrm{Wi}$, measures the impact of shear-thinning on the viscosity. Wi is expressed as: 


$$
\mathrm{Wi}=\frac{\eta_{1}(p, T) \dot{\gamma}}{G}
$$

where $\eta_{1}(p, T)$ is the low shear viscosity, $\dot{\gamma}$ is the shear rate and $G$ is the shear modulus of the lubricant. Shear-thinning can be said to occur for a given liquid when Wi becomes greater than unity (Bair (2)).

The limiting shear stress number, $L i$, was originally proposed by Habchi and coworkers (Habchi et al. (19)). Although the origin of the phenomenon behind the apparent limitation of the shear stress in highly loaded contacts is still under debate, Li conveniently provides a way to predict its onset and offset. $\mathrm{Li}$ is defined as:

$$
\mathrm{Li}=\frac{\tau_{u}}{\tau_{L}}
$$

where $\tau_{u}$ corresponds to the unbounded value of the shear stress, $\tau$, and $\tau_{L}$ is the limiting shear stress. According to measurements, $\tau_{L}$ rises linearly with pressure with a proportionality coefficient $\Lambda$ characteristic of the material (Bair (2)). Typical values of $\Lambda$ for lubricants vary between 0.03-0.15 (Stahl and Jacobson (21)). Without actual experimental data for the chosen lubricant, a value of 0.05 was assumed based on a comparison with other lubricants detailed in (Stahl and Jacobson (21)). When Li is above unity, it can be said that the limiting-shear-stress regime is reached and the greater the value of $\mathrm{Li}$, the greater the truncation in the value of the shear stress $\tau$ (Habchi et al. (19)).

\section{NUMERICAL METHOD}

\subsection{Model}

Numerical simulations of the cam-follower lubricated contact were performed using a Finite Element model based on the full system approach (Habchi et al. (22)). Modifications were introduced to treat both stationary and transient fully flooded line EHD contacts. From a geometrical point of view, the cam-follower contact was reduced to a line contact owing to the large difference in dimensions along the three directions. In addition, smooth surfaces were assumed. Finally, since the structure and specifics of the model were already thoroughly detailed by the authors in a previous paper (Raisin et al. (14)), only a brief reminder is given hereafter.

The present transient Thermal EHL model relies on the coupling of classical equations and on the use of a quantitative description of the lubricant properties through physics-based constitutive laws (defined in section 1.2). These equations are the transient generalized Reynolds equation (Yang and Wen (23)), the steady-state linear elasticity equations, the transient load balance equation and the transient energy-balance equations (see Appendix A or Raisin et al. (14)). These equations are solved using the usual EHL boundary conditions applied to the cam-follower contact problem. For the generalized Reynolds equation, atmospheric pressure is assumed at the boundaries of the fluid domain $\Omega$ defined for $x \in\left[-5.4 b_{\text {ref }}, 5.4 b_{\text {ref }}\right], b_{\text {ref }}$ being the Hertzian half-width at the reference instant, $t_{\text {ref }}$. Symmetry of the fluid domain is mandatory here because cam-follower contacts experience reversal of entrainment during operation. Also, the free boundary problem arising the exit of the contact is handled by applying the penalty method (Habchi et al. (22)). As for the linear elasticity part, the pressure distribution obtained from the Reynolds equation is used as a normal pressure load boundary condition on the contact surface. Null displacements are assumed on the bottom surface of the $60 b_{\text {ref }} \times 60 b_{\text {ref }}$ sized elastic domain, while side surfaces are left free. Finally, for the thermal part, conditional relations are prescribed at the side boundaries of the solid and lubricant domains such that matter entering the contact does so at ambient temperature $T_{0}$ (Raisin et al. (14)). Finally, the continuity of the heat flux 
and temperature are imposed at the fluid/solid interfaces and zero conductive heat fluxes are assumed at the boundaries far from the contact area (located at a depth of $3 b_{\text {ref }}$ ).

\subsection{Procedure}

All the equations and associated boundary conditions were implemented into COMSOL MULTIPHYSICS 4.4 commercial software in their dimensionless form. The nondimensionalization was performed using the following quantities:

$$
\begin{gathered}
\bar{x}=\frac{x}{b_{\mathrm{ref}}}, \bar{z}=\left\{\begin{array}{l}
z / h, \quad \text { in the lubricant } \\
z / b_{\mathrm{ref}} \text { in the solids }
\end{array}\right\}, \bar{t}=\frac{t u_{m_{\mathrm{ref}}}}{b_{\mathrm{ref}}} \\
R_{t}=\frac{R(t)}{R_{\mathrm{ref}}}, W_{t}=\frac{w(t)}{w_{\mathrm{ref}}}, U_{m t}=\frac{u_{2}(t)-u_{1}(t)}{u_{m_{\mathrm{ref}}}} \\
\bar{p}(\bar{x}, \bar{t})=\frac{p(\bar{x}, \bar{t})}{p_{h_{\mathrm{ref}}}}, H(\bar{x}, \bar{t})=\frac{h(\bar{x}, \bar{t}) R_{\mathrm{ref}}}{b_{\mathrm{ref}}}, \bar{\rho}=\frac{\rho(p, T)}{\rho_{0}}, \bar{\eta}=\frac{\eta(p, T, \tau)}{\eta_{0}}
\end{gathered}
$$

where the superscript ()$_{\text {ref }}$ denotes the reference instant, $t_{\text {ref. }}$. Since the dimensions of the camfollower contact, symbolized by the Hertzian half-width $b(t)$, changes significantly over a cycle (up to a factor of five in the system under consideration), a special attention must be paid on the choice of $t_{\text {ref. }}$. Here, a proper scaling of the model was ensured by choosing the camshaft angle $\theta=172^{\circ}$, which gives the largest contact half-width, as the reference instant. As in Habchi's work (Habchi et al. (22)), the meshing of all geometric components is tailored towards the nature of the transient TEHL problem. That is, the mesh is always finer in the central area of the contact where additional precision is required owing to the sharper solution gradients that are encountered (Habchi et al. (22)).

The numerical procedure for the simulation of transient Thermal EHL (TEHL) problems was already presented in (Raisin et al. (14)) and basically consists of two steps. First, a full stationary TEHL simulation is performed using the operating conditions at $t=0 \mathrm{~s}$. The solution then serves as an initial guess for the transient simulation. From a computational point of view, the differential-algebraic system, formed by equations (A.1) and (A.5)-(A.7) in Appendix A, is solved using a variable step size variable order backward differentiation formula scheme. Time steps are automatically adjusted to satisfy specified tolerance of 0.001 and $10^{-5}$ on the relative and absolute errors, respectively. At each time step, the fully coupled non-linear system is linearized using a Newton-Raphson procedure and solved using a monolithic approach. A transient non-Newtonian TEHL simulation (reference case) contains about 69261 degrees of freedom and 3395 time steps, and lasts about 10 hours and 20 minutes on a computer equipped with an Intel Xeon E5-2643 processor.

In order to analyze the respective contribution of shear-thinning, thermal softening and transient effects to the performance of the cam-follower contact, different types of simulation were performed:

- Reference: all three of the aforementioned effects are considered. The "reference" case corresponds to a transient non-Newtonian TEHL simulation.

- Thermal off: thermal effects are disabled. The "thermal off" case corresponds to a transient non-Newtonian isothermal EHL simulation.

- Shear-thinning off, thermal off: both thermal and shear-thinning effects are disabled. The "Shear-thinning off, thermal off" case corresponds to a transient Newtonian isothermal simulation. 
- Transient off: transient effects are disabled. The "transient off" case corresponds to a quasisteady non-Newtonian TEHL simulation.

In the upcoming section, the influence of each effect is deduced by direct comparisons between the results of these four types of simulation.

\section{RESULTS AND DISCUSSION}

\subsection{Cycle analysis}

As a first step towards an in-depth analysis of the cam-follower contact, the evolution of the characteristic numbers (see section 1.3) during a full cycle is presented in Fig. 3. This cycle is composed of four distinct parts, each related to a specific area of the cam contour. These parts are defined as a function of the camshaft angle, $\theta$, as the opening flank (from $\theta=16^{\circ}$ to $\theta=122^{\circ}$ ), the nose (from $\theta=$ $122^{\circ}$ to $\theta=158^{\circ}$ ), the closing flank (from $\theta=158^{\circ}$ to $\theta=255^{\circ}$ ) and the back of the cam (from $\theta=255^{\circ}$ to $\theta=16^{\circ}$ ). As can be seen on Fig. 3, the contact is subjected to severe sliding conditions, SRR being above 2 for the most part. Consequently, it is not surprising to find large values for the Weissenberg and Nahme-Griffith numbers (around 2000 for Wi and between 200 and 30000 for $\mathrm{Na}$ ). Shear thinning and thermal softening are therefore likely to significantly affect the response of the cam-follower contact. In fact, these effects even seem strong enough to keep the shear stress in the contact below the limiting shear stress value (Li remains almost always below unity). From a modeling point of view, this detail is extremely important. It implies that the use of limiting shear stress models, classically used to truncate shear stresses exceeding the limiting value, is unnecessary in the present case. Finally, the interest in relying on characteristic numbers is not limited to the estimation of their respective values. Indeed, direct comparisons between these numbers can also provide crucial information. For instance, the estimation of $\mathrm{Na} / \mathrm{Wi}^{2}$ or $\mathrm{Wi} / \mathrm{Na}^{2}$ can help to assess the dominance of thermal softening over shear-thinning or vice-versa (Bair (2)). Here, neither of these two effects can be strictly said to dominate at any point of the cycle, both ratios remaining below unity. Nonetheless, large differences between $\mathrm{Na}$ and Wi during portions of the cycle let us suppose that one effect may play a more prominent role than the other. A perfect illustration of that, the outstanding influence of thermal effects during the transitions between the opening flank and the nose (around $\theta=125^{\circ}$ ) and between the nose and the closing flank (around $\theta=155^{\circ}$ ) where zero entrainment velocity conditions are encountered, is discussed in the next section.

\subsection{Friction and film thickness}

In this section, the respective influence of shear thinning, thermal softening and transient effects on the performance of the cam-follower contact is further analyzed. Results obtained with the four different modeling hypotheses (defined in section 2.2) for the friction coefficient, $C_{f}$, and the central and minimum film thicknesses, $h_{c}$ and $h_{m}$, are reported on Fig. 4,5 and 6, respectively. The conclusions that can be drawn from these results are rather striking. Indeed, the first unexpected finding concerns the influence of transient effects. Overall, these effects tend to smooth the response of the contact to the imposed level of dynamics. Their influence is slightly more noticeable on the film thickness (Fig. 5 and 6) than on the friction coefficient (Fig. 4), especially during the second ZEVs conditions (camshaft angles around $\theta=155^{\circ}$ ) but remain quantitatively negligible compared to the two other effects. Such a result is particularly surprising as at the considered engine rotational speed, the contact operating conditions are completely dictated by the dynamics of the valve train system. For the friction coefficient, another unexpected finding is the moderate impact of shear thinning effects. From 
a comparison between the Isothermal Newtonian and non-Newtonian simulations on Fig. 4, this impact can be quantified as a friction reduction ranging averaging between 28 and $45 \%$. On the opposite, thermal effects almost completely control the friction response of the cam-follower contact, as evidenced by the differences in the predicted $C_{f}$ between the reference and the isothermal simulations. In practice, the high loads and velocities in the ICEs of racing cars combined with SRR larger than two generates tremendous amount of heat through viscous shearing. Around $\theta=70^{\circ}, 125^{\circ}$ and $155^{\circ}$, the friction reduction induced by viscous heating reaches more than an order of magnitude.

For the film thickness, the picture is quite different. Shear-thinning effects account for the response of the contact over most of the cycle. According to Fig. 5 and 6, the Isothermal Newtonian simulation predicts central and minimum film thicknesses, $h_{c}$ and $h_{m}$, approximately twice larger compared to the Isothermal Non-Newtonian simulation. Again, in practice, the high sliding velocities, $u_{s}$, prevailing in the contact produce shear stresses at the inlet larger than the shear modulus of the lubricant. These shear stresses cause the lubricant viscosity to drop locally, resulting in a decrease of the central and minimum film thicknesses. When the nose of the cam enters (around $\theta=125^{\circ}$ ) and exits (around $\theta=$ $155^{\circ}$ ) the contact, however, thermal effects start to completely dominate the other contributions. During these transitions, ZEV conditions are encountered. In agreement with results from other authors (Messe and Lubrecht (12), Wang and Yang (13)), the isothermal simulations predict a drastic decrease of the central film thickness, especially when the shear-thinning behavior of the lubricant is taken into account. In these simulations as well as in reality, a lubricating film is maintained between the solid surfaces by the squeeze film effect, despite the absence of entrainment (Dowson et al. (7), Glovnea and Spikes (24)). On the opposite, the reference simulation, which includes thermal effects, predicts a much larger central film thickness during the reversals of entrainment (Fig. 5). This large difference is also observed for the minimum film thickness, except for a very short period of time during the second reversal of entrainment where the predicted values are close (Fig. 6). Such a result is particularly important, as in practice, the ZEV conditions are often regarded as the most critical part of the camfollower contact cycle. The underlying phenomenon, known as the thermal viscosity wedge (Fogg (25), Cameron (26)), is presented and discussed in the following section.

\subsection{Transient thermal dimple around the ZEV conditions}

As already reported by different authors in transient (Wang and Yang (13)) or stationary (Guo et al. (27), Bruyere et al. (28)) contact configurations, ZEV/infinite sliding conditions can give rise to dimple-shaped films of lubricant through a mechanism referred to as "thermal viscosity wedge". In stationary conditions, this mechanism occurs as follows: because surface velocities are equal and opposite, the usual definition of contact inlet and outlet no longer applies. In fact, both coexist on each side of the contact. At the inlets, lubricant at ambient temperature is dragged into the contact by one of the moving surfaces. It then gets heated while being entrained through the pressurized region, before exiting the contact at the outlets. A stationary recirculation area, where very high temperatures are reached, forms within the pressurized region, in-between the two 'colder' fluid layers. As a result from this differential heating, a viscosity gradient is created across the film thickness which, in turn, modifies the pressure profile. Figure $7 a$ to $7 f$ illustrates the occurrence of the thermal viscosity wedge mechanism in the dynamic cam-follower contact around the first reversal of entrainment. At a camshaft angle $\theta=117^{\circ}$ (Fig. 7a), the cam (upper surface) and follower (lower surface) move in opposite directions with different velocities in the contact reference system $\left(u_{2}=14.35 \mathrm{~m} \cdot \mathrm{s}^{-1}\right.$ and $u_{1}=-10.06$ $m \cdot s^{-1}$ giving $u_{m}=2.15 m \cdot s^{-1}$ ). 
In practice, an indication of the contact 'inlet' and 'outlet' is given by the presence of a constriction at the right side of Fig. 7a. As the camshaft rotates, the contact approaches the ZEV condition. Temperature rises significantly in the mid plane near the 'outlet' of the contact, where a thermal dimple forms (Fig. 7b). The dimple then grows as the maximum value of the transverse temperature gradient intensifies and progressively shifts toward the center of the contact as the entrainment velocity decreases $\left(u_{m}=0.47 \mathrm{~m} \cdot \mathrm{s}^{-1}\right.$ on Fig. 7c). Between camshaft angles $\theta=123^{\circ}$ (Fig. 7c) and $\theta=126^{\circ}$ (Fig. $7 d)$, ZEV conditions are encountered. The entrainment direction changes as the velocity of the cam becomes lower than the velocity of the follower $\left(u_{2}=11.91 \mathrm{~m} \cdot \mathrm{s}^{-1}\right.$ and $u_{1}=-12.54 \mathrm{~m} \cdot \mathrm{s}^{-1}$ giving $u_{m}=-$ $0.32 \mathrm{~m} \cdot \mathrm{s}^{-1}$ at $\theta=126^{\circ}$ ). Accordingly, the dimple moves toward the new contact outlet (Fig. $7 \mathrm{~d}-\mathrm{e}$ ). It then progressively disappears as the entrainment velocity increases (Fig. 7e-f), reaching up to $u_{m}=-1.72 \mathrm{~m} / \mathrm{s}$ at a camshaft angle $\theta=132^{\circ}$. Along with the transient evolution of the dimple, the particularity of the cam-follower contact in the ICE of racing cars lies in the intensity of the temperature gradient and generated lift. Close to the ZEV condition (between Fig. 7c and Fig. 7d), lubricant temperature rises above $350^{\circ} \mathrm{C}$ in the mid-plane while remaining close to $140^{\circ} \mathrm{C}$ at the fluid/solid interfaces. As a result, the thermal viscosity wedge mechanism produces a substantial lift that increases the central film thickness by factor of four (from $h_{c}=40 \mathrm{~nm}$ to $h_{c}=169 \mathrm{~nm}$ at $\theta=123^{\circ}$ in Fig. 5) and the minimum film thickness by a factor of two and a half (from $h_{m}=17 \mathrm{~nm}$ to $h_{c}=46 \mathrm{~nm}$ at $\theta=125^{\circ}$ in Fig. 6) when compared to the isothermal non-Newtonian configuration.

Finally, another interesting aspect is the duration of the lift. In contrast with the squeeze film mechanism which was shown to influence the cam-follower contact film thickness over a very limited angular portion of the cycle (Messe and Lubrecht (12)), the thermal viscosity wedge mechanism acts for a relatively long duration. The difference in duration between these two mechanisms is clear when looking at the central pressure variation close to the ZEV condition (Fig. 8). In the absence of thermal effects (see the Isothermal Newtonian and non-Newtonian simulations in Fig. 8.), the additional lift which maintains a non-zero film thickness during the ZEV condition manifest itself in the form of a pressure wave (Messe and Lubrecht (12)). The wave, originating from the squeeze-film mechanism, is generated at the contact outlet (here between $\theta=123^{\circ}$ and $\theta=126^{\circ}$ ) and transits through the contact at the entrainment velocity, hence the delayed apparition at the contact center (Glovnea and Spikes (24)). As seen on Figure 8 , both the amplitude (about $80 \mathrm{MPa}$ peak) and duration ( $2^{\circ}$ of camshaft angle) of the "positive" part of wave, which is actually providing the lift, are limited. On the contrary, the contribution of thermal effects to pressure around the ZEV condition is far more intense and no longer appears as a wave (see the Reference and Quasisteady TEHL simulations in Fig. 8). Evidence of the influence of the thermal viscosity wedge can be observed between $\theta=119^{\circ}$ and $\theta=129^{\circ}$ which is approximately five times the duration of the lift due to the squeeze-film mechanism. Transient effects slightly modify the shape of the thermal contribution to pressure but have negligible impact on its intensity (about $200 \mathrm{MPa}$ ). The longer duration as well as the greater intensity basically explains why thermal effects completely dominate the film thickness response of the cam-follower contact around the ZEV conditions.

\section{CONCLUSION}

This article dealt with the lubrication of the cam-follower contacts found in the internal combustion engines (ICEs) of racing cars. Considering line contacts and smooth surfaces, dimensionless number analysis and numerical simulations were used to quantify the respective influence of shear- 
thinning, thermal softening and transient effects on the contact performance and ultimately to provide ideas for lubricant optimization.

Regarding the frictional response, thermal softening was found to completely overwhelm the other effects. In fact, thermal effects in these contacts are so important that omitting to consider their influence may lead to an error of at least an order of magnitude on the predicted friction coefficient.

As for film thickness, shear-thinning was identified as the prominent factor for the most part of the cycle, causing an average reduction by half of both the central and the minimum value for the retained lubricant.

Yet, for two specific portions of the cycle corresponding to the transition toward and away from the cam nose, thermal effects were also found to completely dominate the other effects. During these transitions, the contact experiences a reversal of entrainment and infinite SRR conditions. As a result, tremendous amount of heat is produced in the lubricant through viscous shearing. Temperature rises dramatically in the mid-plane of the film thickness while remaining close to ambient near the fluid/solid interfaces. Immediately before the reversal of entrainment, an intense transverse temperature gradient forms near the 'outlet' of the contact. Additional lift is generated through the so-called "thermal viscosity wedge mechanism", giving rise to a dimple-shaped film. The dimple then transits through the cam-follower contact as the direction of entrainment changes.

The thermal viscosity wedge mechanism is of crucial importance as it significantly increases the central and minimum film thickness (and thus the load carrying capacity) of the contact at an instant of the cycle which would otherwise be extremely severe.

In addition, this article showed that, contrary to previous belief, this thermal mechanism completely outperforms the squeeze film effect, lasting longer and contributing with a much greater intensity. Also, transient effects were found to only play a minor role in smoothing the dynamic variations of the contact conditions.

To sum up, this article provided a clearer understanding of the effects that govern the performance of cam-follower contacts in the ICEs of racing cars. Shear-thinning and thermal softening were clearly identified as the key effects, as long as these contacts operate in the Thermal EHL regime.

\section{ACKNOWLEDGMENT}

The authors are deeply grateful to Total Marketing \& Services for their financial support.

\section{NOMENCLATURE}

$a$
$a_{v}$
$A_{1}, A_{2}, B_{1}, B_{2}, C_{1}, C_{2}$
$b$
$c$
$C_{f}$
$c_{i}$
$E^{\prime}$

Parameter of the modified Carreau-Yasuda law (-)

Thermal expansivity defined for volume linear with temperature $\left(\mathrm{K}^{-1}\right)$. Used in the Tait equation of state.

Parameters of the WLF model

Hertzian half-width ( $m$ )

Specific heat capacity of the lubricant $\left(\mathrm{J} . \mathrm{kg}^{-1} \cdot \mathrm{K}^{-1}\right)$

Friction coefficient (-)

Specific heat capacity of solid $\Omega_{\mathrm{i}}\left(\mathrm{J} \cdot \mathrm{kg}^{-1} \cdot \mathrm{K}^{-1}\right)$

Reduced elastic modulus $(\mathrm{Pa}) \cdot E^{\prime}=2 /\left(\left(1-v_{1}^{2}\right) / E_{1}+\left(1-v_{2}^{2}\right) / E_{2}\right)$ 


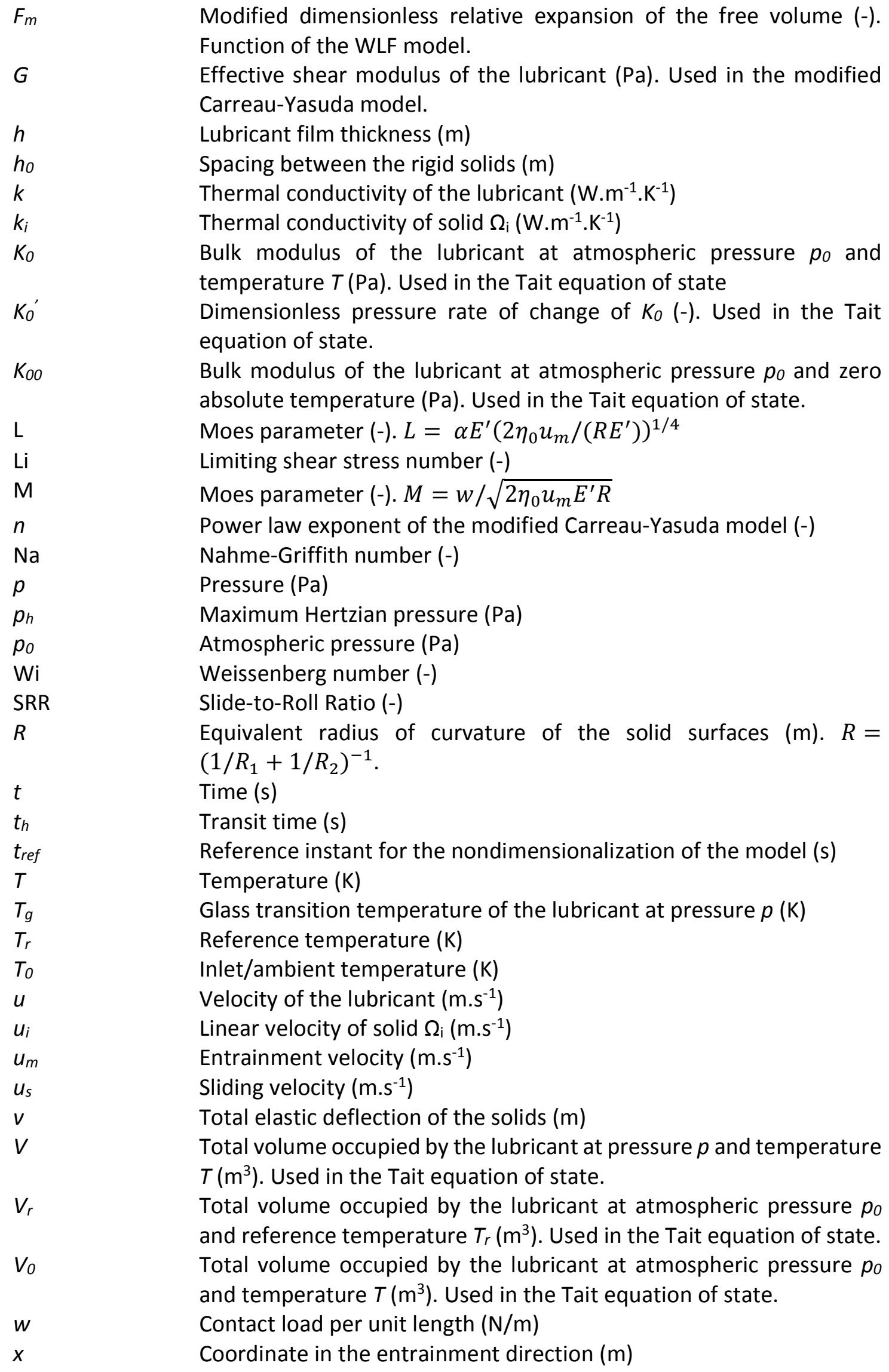

Limiting shear stress number (-)

Moes parameter (-). $M=w / \sqrt{2 \eta_{0} u_{m} E^{\prime} R}$

Power law exponent of the modified Carreau-Yasuda model (-)

Nahme-Griffith number (-)

Pressure $(\mathrm{Pa})$

Maximum Hertzian pressure ( $\mathrm{Pa})$

Atmospheric pressure $(\mathrm{Pa})$

Weissenberg number (-)

Slide-to-Roll Ratio (-)

Equivalent radius of curvature of the solid surfaces $(\mathrm{m}) . R=$ $\left(1 / R_{1}+1 / R_{2}\right)^{-1}$.

Time (s)

Transit time (s)

Reference instant for the nondimensionalization of the model ( $s$ )

Temperature (K)

Glass transition temperature of the lubricant at pressure $p(\mathrm{~K})$

Reference temperature $(\mathrm{K})$

Inlet/ambient temperature $(\mathrm{K})$

Velocity of the lubricant $\left(\mathrm{m} . \mathrm{s}^{-1}\right)$

Linear velocity of solid $\Omega_{\mathrm{i}}\left(\mathrm{m} . \mathrm{s}^{-1}\right)$

Entrainment velocity $\left(\mathrm{m} . \mathrm{s}^{-1}\right)$

Sliding velocity $\left(\mathrm{m} . \mathrm{s}^{-1}\right)$

Total elastic deflection of the solids $(\mathrm{m})$

Total volume occupied by the lubricant at pressure $p$ and temperature $T\left(\mathrm{~m}^{3}\right)$. Used in the Tait equation of state.

Total volume occupied by the lubricant at atmospheric pressure $p_{0}$ and reference temperature $T_{r}\left(\mathrm{~m}^{3}\right)$. Used in the Tait equation of state. Total volume occupied by the lubricant at atmospheric pressure $p_{0}$ and temperature $T\left(\mathrm{~m}^{3}\right)$. Used in the Tait equation of state.

Contact load per unit length $(\mathrm{N} / \mathrm{m})$

Coordinate in the entrainment direction $(\mathrm{m})$ 
Temperature viscosity coefficient of the lubricant $\left(\mathrm{K}^{-1}\right)$. Used in the Nahme-Griffith number.

$\beta_{K}$

$\Lambda$

$\eta$

$\eta g$

$\eta_{0}$

$\eta_{1}$

$\eta_{2} / \eta_{1}$

$\dot{\gamma}$

$\Omega$

$\Omega_{i}$

$\rho$

$\rho_{i}$

$\rho_{r}$

$\rho_{0}$

$\tau$

$\tau_{L}$

$\tau_{u}$

$\theta$

()$_{\mathrm{c}}$

()ref

Temperature coefficient of $K_{0}\left(\mathrm{~K}^{-1}\right)$. Used in the Tait equation of state. Pressure coefficient of the limiting shear stress $\tau_{L}\left(\mathrm{~Pa}^{-1}\right)$

Generalized Newtonian viscosity at pressure $p$ and temperature $T$ (Pa.s)

Viscosity of the lubricant at the glass transition temperature $T_{g}$ and atmospheric pressure $p_{0}$ (Pa.s)

Viscosity of the lubricant at atmospheric pressure $p_{0}$ and inlet/ambient temperature $T_{0}$ (Pa.s)

Newtonian/Low shear viscosity of the lubricant at pressure $p$ and temperature $T$ (Pa.s). Used in the modified Carreau-Yasuda model.

Relative viscosity of the lubricant at the second Newtonian plateau (). Used in the modified Carreau-Yasuda model.

Shear rate $\left(\mathrm{s}^{-1}\right)$

Fluid/lubricant domain

Solid domains

Density of the lubricant at pressure $p$ and temperature $T\left(\mathrm{~kg} \cdot \mathrm{m}^{-3}\right)$

Density of solid $\Omega_{i}\left(\mathrm{~kg} \cdot \mathrm{m}^{-3}\right)$

Density of the lubricant at atmospheric pressure $p_{0}$ and temperature $T_{r}\left(\mathrm{~kg} . \mathrm{m}^{-3}\right)$

Density of the lubricant at atmospheric pressure $p_{0}$ and inlet/ambient temperature $T_{0}\left(\mathrm{~kg} \cdot \mathrm{m}^{-3}\right)$

Shear stress $(\mathrm{Pa})$

Limiting shear stress $(\mathrm{Pa})$ at pressure $p$. Used in the limiting shear stress number.

Unbounded value of the shear stress ( $\mathrm{Pa})$. Used in the limiting shear stress number.

Camshaft angle $\left({ }^{\circ}\right)$

Value at the contact center

Value at the reference instant $t_{\text {ref }}$ 


\section{APPENDIX A: Equations of the TEHD lubricated contact problem}

As mentioned in section 2.1, the transient Thermal Elastohydrodynamic modeling of lubricated contact relies on the coupling of four different equations. These equations are described in this appendix.

The generalized Reynolds equation governs the flow inside the fluid domain $\Omega$. Originally proposed by Yang and Wen (Yang and Wen (22)), the equation applied to transient line contact problems can be written in the form:

$$
\frac{\partial}{\partial x}\left[\left(\frac{\rho}{\eta}\right)_{e} \frac{\partial p(x, t)}{\partial x}\right]-\frac{\partial}{\partial x}\left(\rho^{*}\right)-\frac{\partial}{\partial t}\left(\rho_{e}\right)=0
$$

where $p(x, t)$ represents the instantaneous pressure at any given point in the fluid. Other quantities in eq. (A.1) read:

$$
\begin{aligned}
& \left(\frac{\rho}{\eta}\right)_{e}=\frac{\eta_{e}}{\eta_{e}^{\prime}} \rho_{e}^{\prime}-\rho_{e}^{\prime \prime} \\
& \rho^{*}=\rho_{e}^{\prime} \eta_{e}\left(u_{2}(t)-u_{1}(t)\right)-\rho_{e} u_{1}(t)
\end{aligned}
$$

where $u_{1}$ and $u_{2}$ are the velocities of the contacting bodies along the $x$ direction and variables with the superscript ()$_{e}$ represent equivalent terms taking into account the variations of the lubricant properties across the film thickness. These terms are expressed as:

$$
\begin{aligned}
& \rho_{e}=\int_{0}^{h(x, t)} \rho(p, T) d z \\
& \rho_{e}^{\prime}=\int_{0}^{h(x, t)} \rho(p, T)\left(\int_{0}^{z} \frac{1}{\eta(p, T, \tau)} d z^{\prime}\right) d z \\
& \rho_{e}^{\prime \prime}=\int_{0}^{h(x, t)} \rho(p, T)\left(\int_{0}^{z} \frac{z^{\prime}}{\eta(p, T, \tau)} d z^{\prime}\right) d z \\
& \frac{1}{\eta_{e}}=\int_{0}^{h(x, t)} \frac{1}{\eta(p, T, \tau)} d z \\
& \frac{1}{\eta_{e}^{\prime}}=\int_{0}^{h(x, t)} \frac{z}{\eta(p, T, \tau)} d z
\end{aligned}
$$

where $\rho(p, T)$ and $\eta(p, T, \tau)$ represent the density and generalized viscosity, respectively. $h(x, t)$ is the instantaneous film thickness at any point of the fluid domain $\Omega$ defined as: 


$$
h(x, t)=h_{0}(t)+\frac{x^{2}}{2 R(t)}-v(x, t)
$$

It is composed of $h_{0}(t)$, the instantaneous spacing between the underformed solids, $R(t)$, the instantaneous equivalent radius of curvature of the solid surfaces, and $v(x, t)$, the instantaneous total elastic deflection of the solids. This deflection is obtained by solving the linear elasticity equations on a much larger domain representing both solids through the use of the equivalent body theory (Habchi et al. (21)). To complete the EHL coupling, the load balance equation is solved to ensure that the correct external load $w(t)$ is applied to the contact. This equation reads:

$$
\int_{\Omega} p(x, t) d x=w(t)
$$

As for the thermal part, the transient energy-balance equations are used to calculate the temperature distributions in the solids and in the lubricant. Neglecting body forces, heat radiation and heat advection along the $z$ direction, these equations for solids $\Omega_{1}$ and $\Omega_{2}$ read (Raisin et al. (14)):

$$
\rho_{i} c_{i}\left(u_{i}(t) \frac{\partial T}{\partial x}+\frac{\partial T}{\partial t}\right)-\frac{\partial}{\partial x}\left(k_{i} \frac{\partial T}{\partial x}\right)-\frac{\partial}{\partial z}\left(k_{i} \frac{\partial T}{\partial z}\right)=0, \quad i=1,2
$$

where $\rho_{i}, c_{i}$ and $k_{i}$ are the density, heat capacity and thermal conductivity of solid $\Omega_{i}$, respectively. In the lubricant, diffusion along the entrainment direction can also be neglected, reducing the transient energy-balance equation to:

$$
\begin{aligned}
\rho c(u(x, z, t) & \left.\frac{\partial T}{\partial x}+\frac{\partial T}{\partial t}\right)-\frac{\partial}{\partial z}\left(k \frac{\partial T}{\partial z}\right) \\
& =-\frac{T}{\rho} \frac{\partial \rho}{\partial T}\left(u(x, z, t) \frac{\partial p}{\partial x}+\frac{\partial p}{\partial t}\right)+\eta(p, T, \tau)\left(\frac{\partial u(x, z, t)}{\partial z}\right)^{2}
\end{aligned}
$$

where the fluid velocity, $u(x, z, t)$, is:

$$
\begin{aligned}
u(x, z, t)=\frac{\partial p}{\partial x} & \left(\int_{0}^{z} \frac{z}{\eta(p, T, \tau)} d z-\frac{\eta_{e}}{\eta_{e}^{\prime}} \int_{0}^{z} \frac{1}{\eta(p, T, \tau)} d z\right) \\
& +\eta_{e}\left(u_{2}(t)-u_{1}(t)\right) \int_{0}^{z} \frac{1}{\eta(p, T, \tau)} d z+u_{1}(t)
\end{aligned}
$$

The first and second right-hand side terms in eq. (A.7) account for the production of heat by compression and shearing, respectively. Equations (A.1)-(A.8) completely define the transient Thermal EHL problem. 


\section{REFERENCES}

(1) Total Competition, "2014 Press kit (in French)".

http://204.92.52.209/Competition/pdf/F1/Total-DP2014F1-FR-EXE.pdf (accessed September24, 2015)

(2) Bair, S. (2007), "High-Pressure Rheology for Quantitative Elasto-hydrodynamics", Elsevier, Burlington, USA, Chap. 4,7,8,10. ISBN: 9780080475301

(3) Mary, C., Philippon, D., Devaux, N., Fillot, N., Laurent, D., Bair, S. and Vergne, P. (2015) "Bridging high pressure rheology and film-forming capacity of polymer-base oil solutions in EHL", Tribology International.

http://dx.doi.org/10.1016/j.triboint.2014.08.006

(4) Björling, M., Habchi, W., Bair, S., Larsson, R. and Marklund, P. (2014), "Friction Reduction in Elastohydrodynamic Contacts by Thin-Layer Thermal Insulation", Tribology Letters, 53, pp. 477-486. http://dx.doi.org/10.1007/s11249-013-0286-8

(5) Habchi, W. (2014), "A numerical model for the solution of thermal elastohydrodynamic lubrication in coated circular contacts", Tribology International, 73, pp. 57-68. http://dx.doi.org/10.1016/j.triboint.2014.01.002

(6) Ciulli, E., Fazzolari, F. and Piccigallo, B. (2014), "Experimental study on circular eccentric camfollower pairs", Proceedings of the Institution of Mechanical Engineers Part J: Journal of Engineering Tribology, 228 (10), pp. 1088-1098.

http://dx.doi.org/10.1177/1350650114529943

(7) Dowson, D., Harrison, P. and Taylor, C. M. (1986), "The Lubrication of Automotive Cams and Followers". Proceedings of the $12^{\text {th }}$ Leeds-Lyon Symposium on Tribology -Mechanisms and Surface Distress, Butterworth, London, 1986, pp. 305-322.

(8) Bedewi, M. A., Dowson, D. and Taylor, C. M. (1986), "Elastohydrodynamic Lubrication of Line Contacts Subjected to Time Dependent Loading with Particular Reference to Roller Bearings and Cams and Followers", Proceedings of the $12^{\text {th }}$ Leeds-Lyon Symposium on Tribology-Mechanisms and Surface Distress, Butterworth, London, 1986, pp. 289-304.

(9) Chong, W. W. F., Teodorescu, M. and Rahnejat, H. (2014), "Mixed thermo-elastohydrodynamic camtappet power loss in low speed emission cycle", International Journal of Engine Research, 15 (2), pp. 153-164.

http://dx.doi.org/10.1177/1468087412461631

(10) Zavos, A. B. and Nikolakopoulos, P. G (2014), "Simulation of piston ring tribology with surface texturing for internal combustion engines", Lubrication Science, 27, pp. 151-176.

http://dx.doi.org/10.1002/ls.1261

(11) Ai, X. and Yu, H. (1989), "A Numerical Analysis for the Transient EHL Process of a Cam-Tappet Pair in I.C. Engine," Journal of Tribology, 111 (3), pp. 413-417.

http://dx.doi.org/10.1115/1.3261940

(12) Messe, S. and Lubrecht, A. A. (2000), "Transient elastohydrodynamic analysis of an overhead cam/tappet contact", Proceedings of the Institution of Mechanical Engineers Part J: Journal of Engineering Tribology, 214 (5), pp. 415-425. 
http://dx.doi.org/10.1243/1350650001543296

(13) Wang, J. and Yang, P. (2003), "A Numerical Analysis for TEHL of Eccentric-Tappet Pair Subjected to Transient Load", Journal of Tribology, 125 (4), pp. 770-779.

http://dx.doi.org/10.1115/1.1576425

(14) Raisin, J., Fillot, N., Dureisseix, D., Vergne, P. and Lacour, V. (2015), "Characteristic times in transient thermal elastohydrodynamic line contacts", Tribology International, 82 (Part B), pp. 472-483. http://dx.doi.org/10.1016/i.triboint.2014.02.022

(15) Vergne, P. and Bair, S. (2014), "Classical EHL Versus Quantitative EHL: A Perspective Part I-Real Viscosity-Pressure Dependence and the Viscosity-Pressure Coefficient for Predicting Film Thickness", Tribology Letters, 54 (1), pp. 1-12.

http://dx.doi.org/10.1007/s11249-014-0302-7

(16) Moes, H. (1992), "Optimum similarity analysis with applications to elastohydrodynamic lubrication", Wear, 159, pp. 57-66.

(17) Mary, C., Philippon, D., Lafarge, L., Laurent, D., Rondelez, F., Bair, S. and Vergne, P. (2013), “New Insight into the Relationship Between Molecular Effects and the Rheological Behavior of PolymerThickened Lubricants Under High Pressure", Tribology Letters, 52 (3), pp. 357-369. http://dx.doi.org/10.1007/s11249-013-0214-y

(18) Bair, S., Mary, C., Bouscharain, N. and Vergne, P. (2013), "An improved Yasutomi correlation for viscosity at high pressure", Proceedings of the Institution of Mechanical Engineers Part J: Journal of Engineering Tribology, 227 (9), pp. 1056-1060.

http://dx.doi.org/10.1177/1350650112474394

(19) Habchi, W., Bair, S. and Vergne, P. (2013), "On friction regimes in quantitative elastohydrodynamics", Tribology International, 58, pp. 107-117.

http://dx.doi.org/10.1016/i.triboint.2012.10.005

(20) Winter, H. H. (1977), "Viscous dissipation in shear flows of molten polymers", Advances in Heat Transfer, 13, pp. 205-67.

(21) Stahl, J. and Jacobson, B. O. (2003), "A non-Newtonian model based on limiting shear stress and slip planes-parametric studies", Tribology International, 36, pp. 801-806. http://dx.doi.org/10.1016/S0301-679x(03)00096-3

(22) Habchi, W., Eyheramendy, D., Vergne, P. and Morales-Espejel, G. (2012), "Stabilized fully-coupled finite elements for elastohydrodynamic lubrication problems", Advances in Engineering Software, 46, pp. 4-18. http://dx.doi.org/10.1016/i.advengsoft.2010.09.010

(23) Yang, P. and Wen, S. (1990), "A Generalized Reynolds Equation for Non-Newtonian Thermal Elastohydrodynamic Lubrication", Journal of Tribology, 112 (4), pp. 631-636.

(24) Glovnea, R. P. and Spikes, H. A. (2002), "Behavior of EHD Films During Reversal of Entrainment in Cyclically Accelerated/Decelerated Motion", Tribology Transactions, 45 (2), pp. 177-184. http://dx.doi.org/10.1080/10402000208982537

(25) Fogg, A. (1946), "Fluid Film Lubrication of Parallel Thrust Surfaces", Proceedings of the Institution of Mechanical Engineers, 155 (1), pp. 49-67.

http://dx.doi.org/10.1243/PIME PROC 194615501102

(26) Cameron, A. (1958), "The viscosity wedge”, ASLE Transactions; 1, pp. 248-253. 
(27) Guo, F., Wong, P. L., Yang, P. and Yagi, K. (2002), "Film Formation in EHL Point Contacts under Zero Entraining Velocity conditions", Tribology Transactions, 45 (4), pp. 521-530. http://dx.doi.org/10.1080/10402000208982583

(28) Bruyere, V., Fillot, N., Morales-Espejel, G. E. and Vergne, P. (2012), "Computational fluid dynamics and full elasticity model for sliding line thermal elastohydrodynamic contacts", Tribology International, 46, pp. 3-13.

http://dx.doi.org/10.1016/i.triboint.2011.04.013

Table 1: Material properties used for the solids

\begin{tabular}{|l|l|l|}
\hline Property & Unit & Value \\
\hline$\rho_{1,2}$ & $\mathrm{~kg} \cdot \mathrm{m}^{-3}$ & 7850 \\
\hline $\mathrm{E}_{1,2}$ & $\mathrm{~Pa}$ & $210 \times 10^{9}$ \\
\hline$v_{1,2}$ & - & 0.3 \\
\hline $\mathrm{C}_{1,2}$ & $\mathrm{~J} \cdot \mathrm{kg}^{-1} \cdot \mathrm{K}^{-1}$ & 470 \\
\hline $\mathrm{k}_{1,2}$ & $\mathrm{~W} \cdot \mathrm{m}^{-1} \cdot \mathrm{K}^{-1}$ & 46 \\
\hline
\end{tabular}


Table 2: Parameters of the Tait, WLF and Carreau-Yasuda model for the retained lubricant, characterized by Mary and coworkers (Mary et al. (3)).

\begin{tabular}{|c|c|c|}
\hline Property & Unit & Value \\
\hline \multicolumn{3}{|c|}{ Tait parameters } \\
\hline$\rho_{r}$ & $\mathrm{~kg} \cdot \mathrm{m}^{-3}$ & 836.8 \\
\hline$K_{0}^{\prime}$ & - & 11 \\
\hline$a_{v}$ & $\mathrm{~K}^{-1}$ & $7.922 \times 10^{-4}$ \\
\hline$T_{r}$ & $\mathrm{~K}$ & 288 \\
\hline$K_{00}$ & $\mathrm{~Pa}$ & $9 \times 10^{9}$ \\
\hline$\beta_{k}$ & $\mathrm{~K}^{-1}$ & $6.18 \times 10^{-3}$ \\
\hline \multicolumn{3}{|c|}{ WLF parameters } \\
\hline$C_{1}$ & - & 15.85 \\
\hline$C_{2}$ & $\mathrm{~K}$ & 22.74 \\
\hline$A_{1}$ & $\mathrm{~K}$ & 652.50 \\
\hline$A_{2}$ & $\mathrm{~Pa}^{-1}$ & $5.63 \times 10^{-11}$ \\
\hline$B_{1}$ & $\mathrm{~Pa}^{-1}$ & $8.33 \times 10^{-9}$ \\
\hline$B_{2}$ & - & -0.51 \\
\hline$T_{g}\left(p_{0}\right)$ & $\mathrm{K}$ & 189.45 \\
\hline$\eta\left(T_{g}\left(p_{0}\right)\right)$ & Pa.s & $10^{-12}$ \\
\hline \multicolumn{3}{|c|}{ Carreau-Yasuda parameters } \\
\hline$G$ & $\mathrm{~Pa}$ & $1.1 \times 10^{4}$ \\
\hline$a$ & - & 0.41 \\
\hline$n$ & - & 0.87 \\
\hline$\mu_{2} / \mu_{1}$ & - & 0.41 \\
\hline \multicolumn{3}{|c|}{ Thermal properties } \\
\hline$k$ & $\mathrm{~W} \cdot \mathrm{m}^{-1} \cdot \mathrm{K}^{-1}$ & 0.114 \\
\hline$c$ & J.kg-1. $\mathrm{K}^{-1}$ & 2247 \\
\hline
\end{tabular}




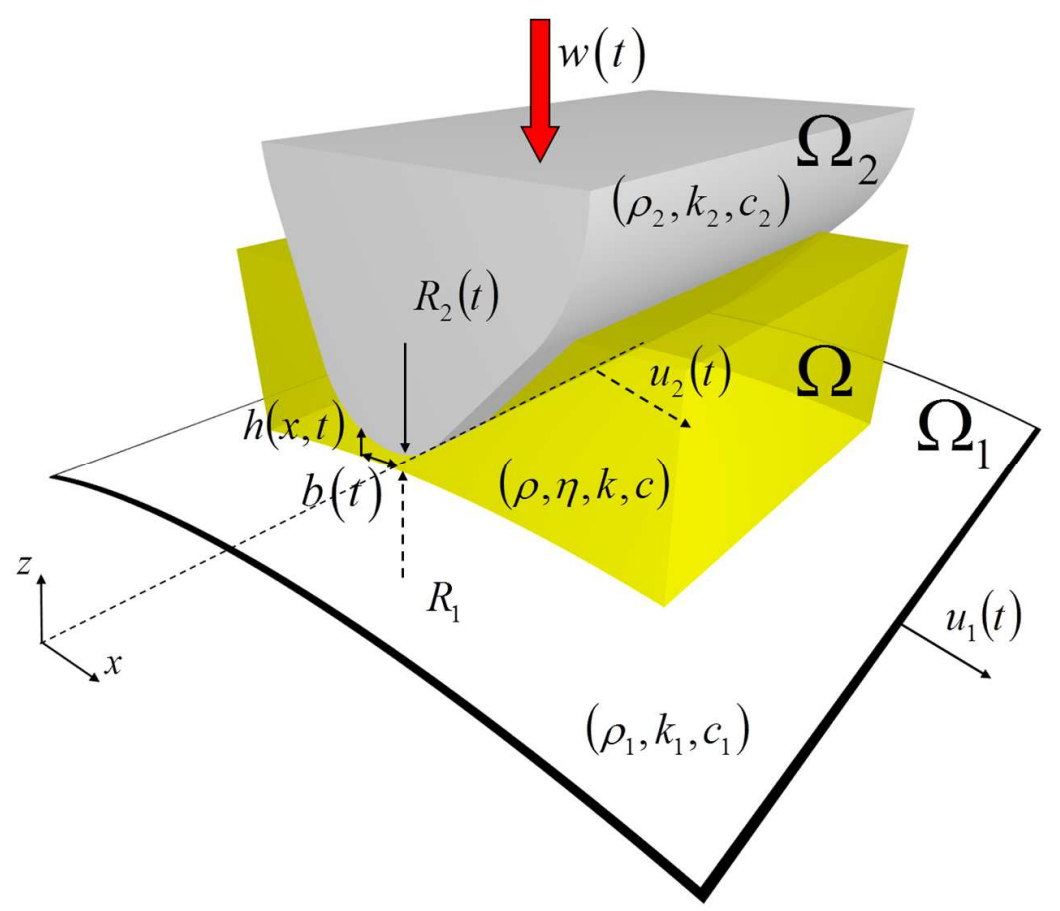

Fig. 1 Schematic representation of a typical lubricated contact between a cam $(\Omega 2)$ and a follower $(\Omega 1) . \Omega$ is the fluid domain.

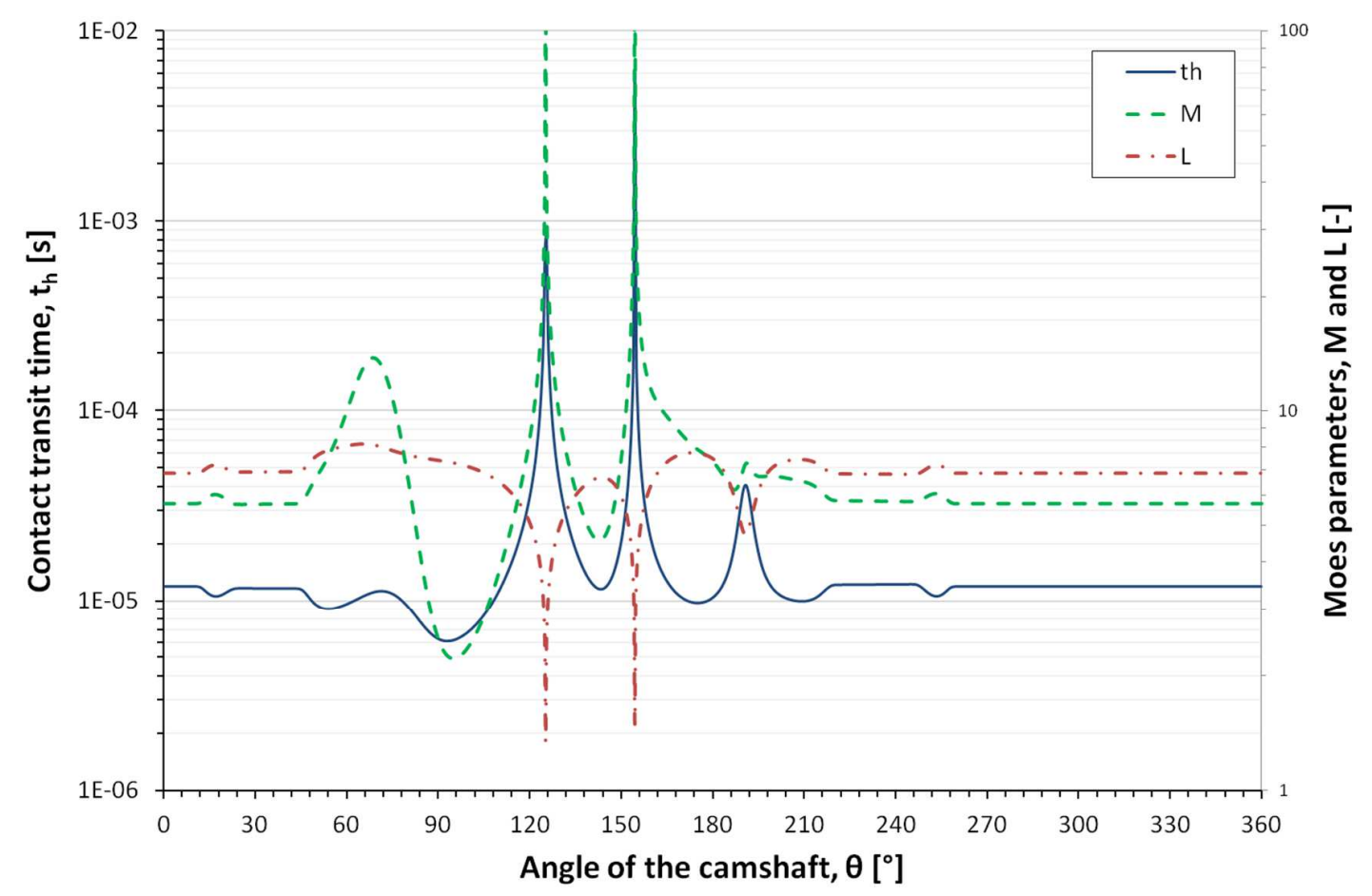

Fig. 2 Variation of the Moes parameters, $M$ and $L$, and of the contact transit time, $t_{h}$, for the camfollower contact over a cycle. Note that $\mathrm{M}$ and $\mathrm{L}$ were computed using the absolute value for the sum of the surface velocities, $u_{1}(t)+u_{2}(t)$, which takes negative values between camshaft angles of $125^{\circ}$ and $155^{\circ}$. 


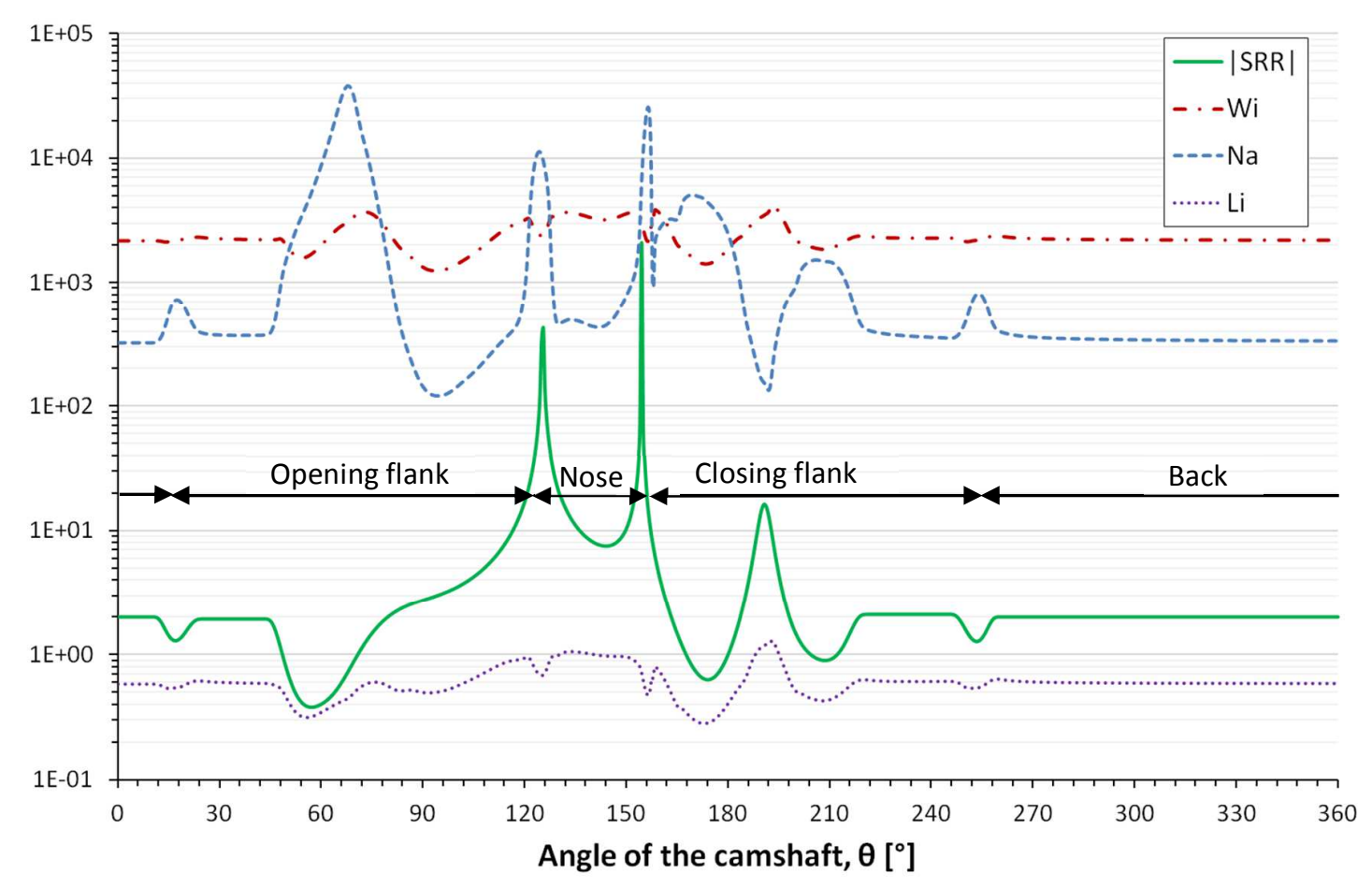

Fig. 3 Variation of the characteristic numbers ( $|S R R|, N a, W i$ and Li) for the cam-follower contact over a cycle obtained from the reference simulation. Here the absolute value of SRR has been used to accommodate the logarithmic scale (SRR values are mostly negative on the angular portion of the cycle corresponding to the nose of the cam).

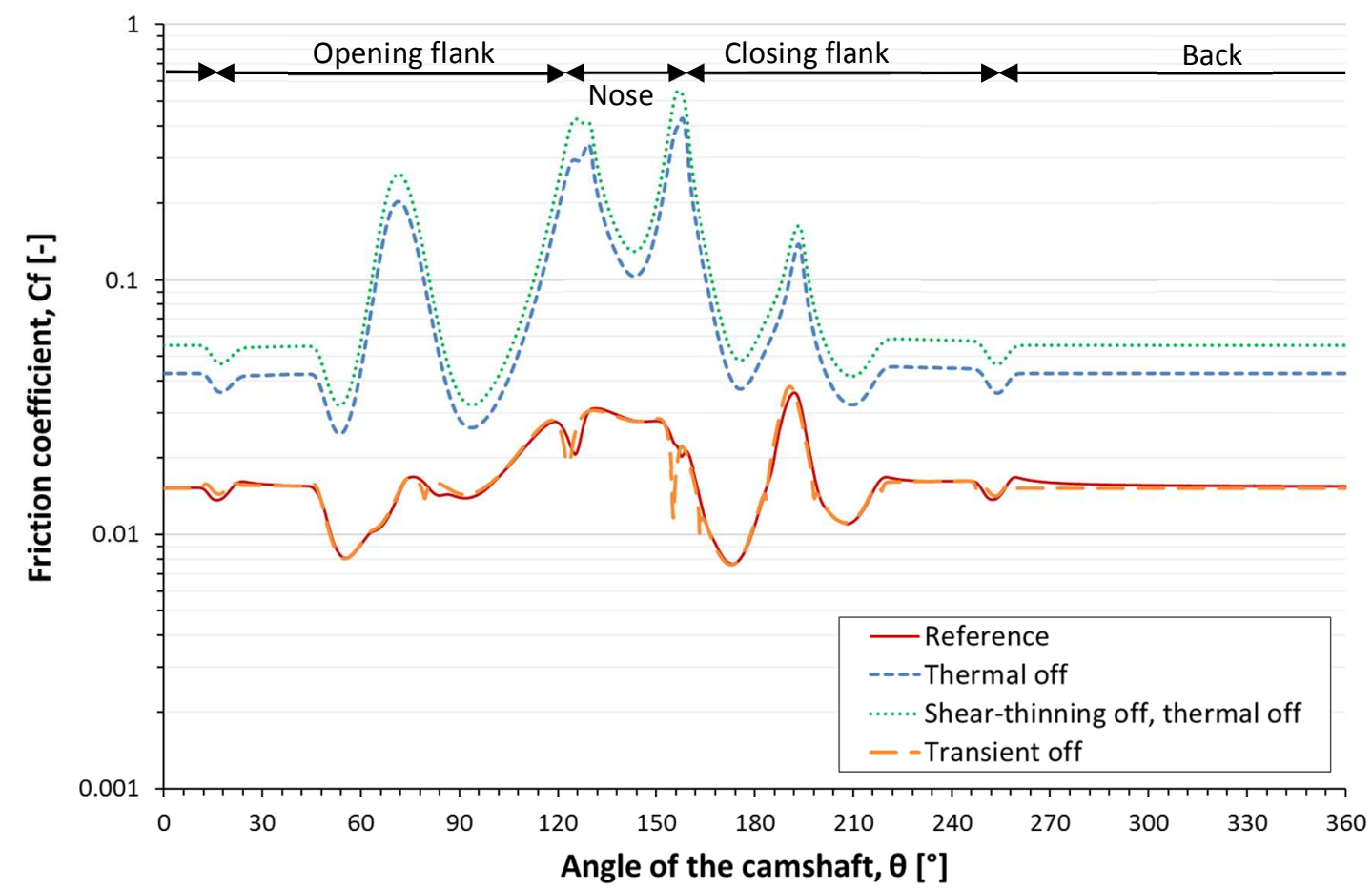

Fig. 4 Variation of the friction coefficient in the cam-follower contact over a full cycle. 


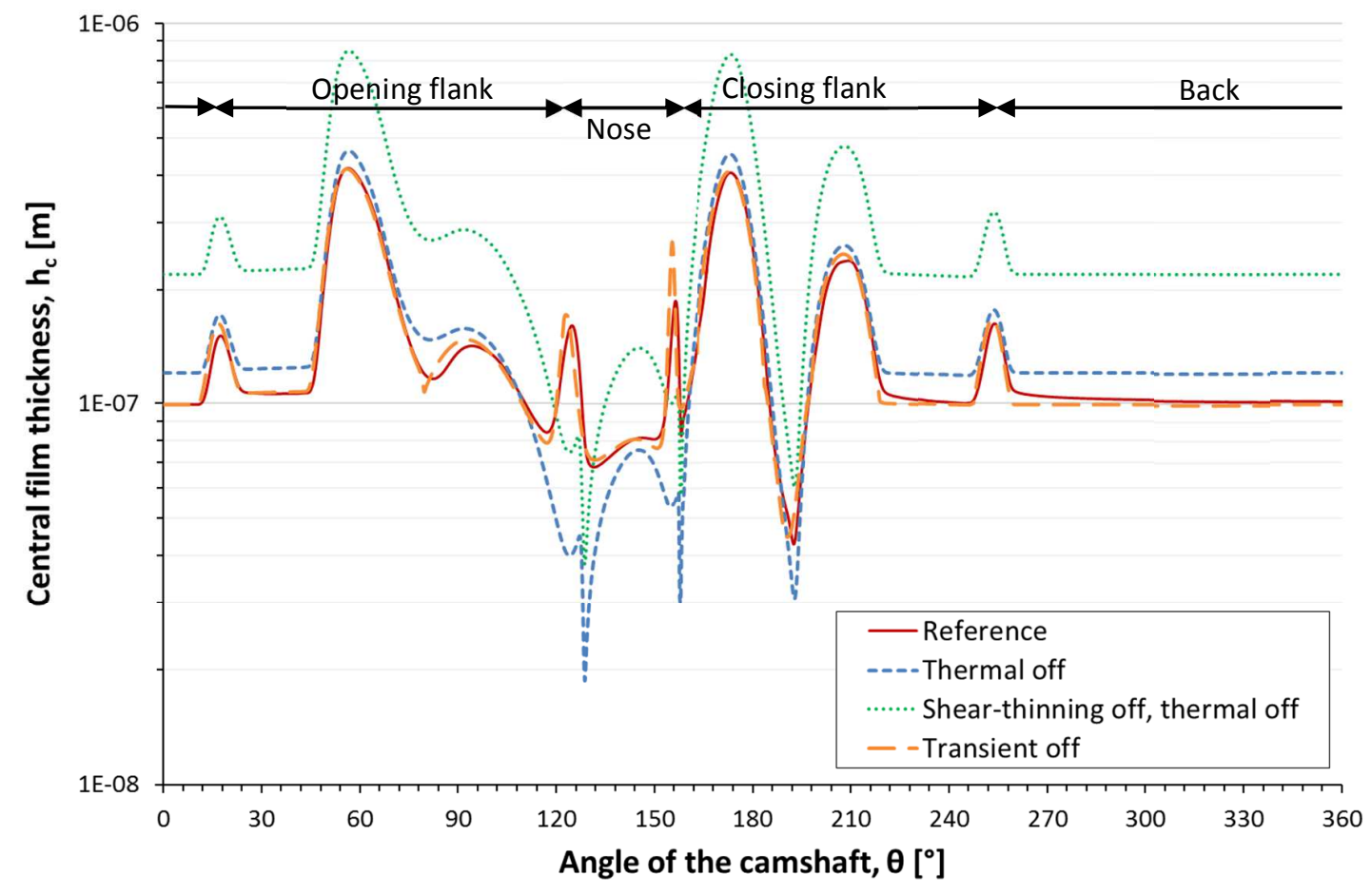

Fig. 5 Variation of the central film thickness in the cam-follower contact over a full cycle.

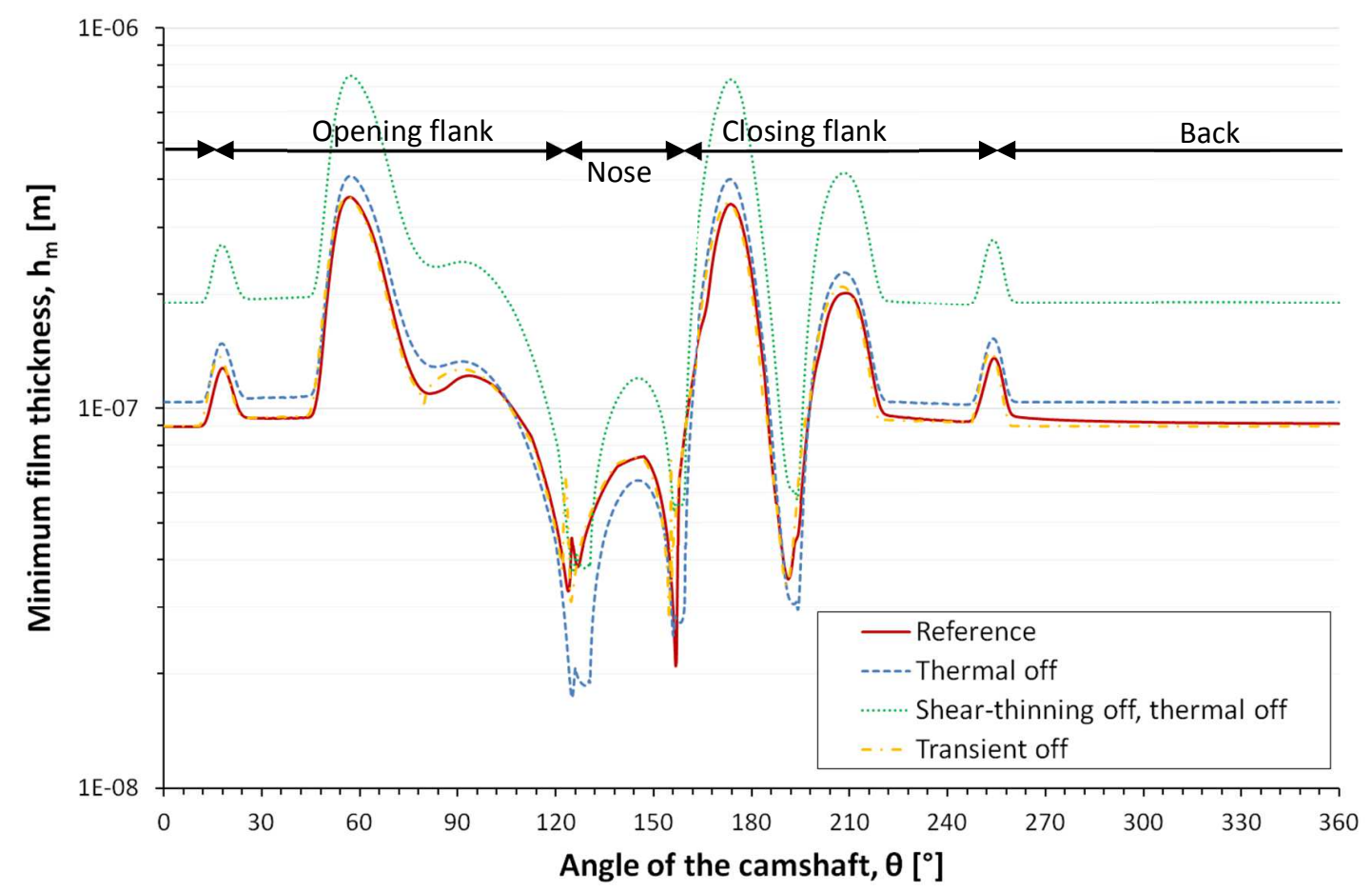

Fig. 6 Variation of the minimum film thickness in the cam-follower contact over a full cycle. 


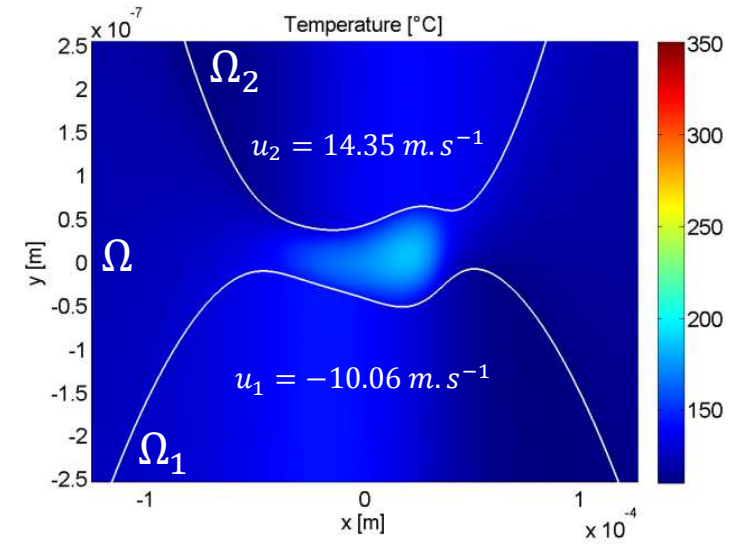

(a)

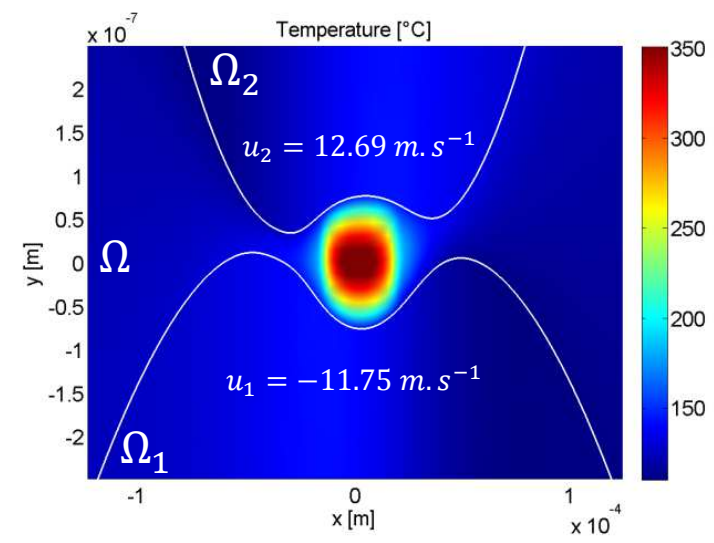

(c)

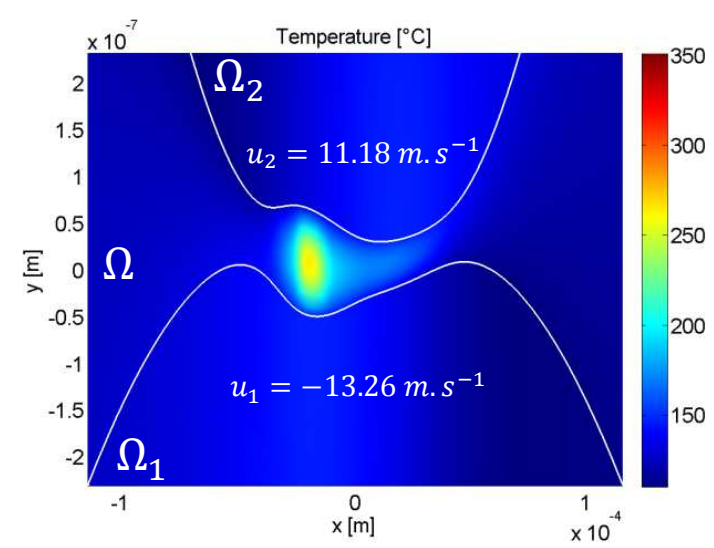

(e)

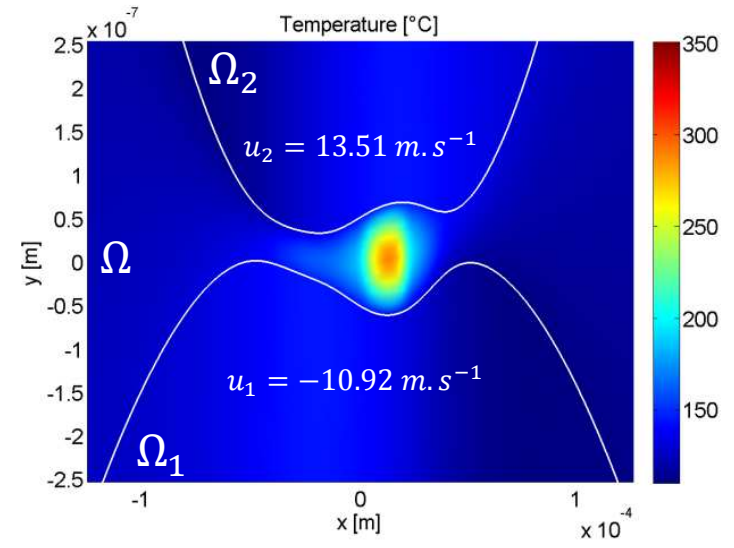

(b)



(d)

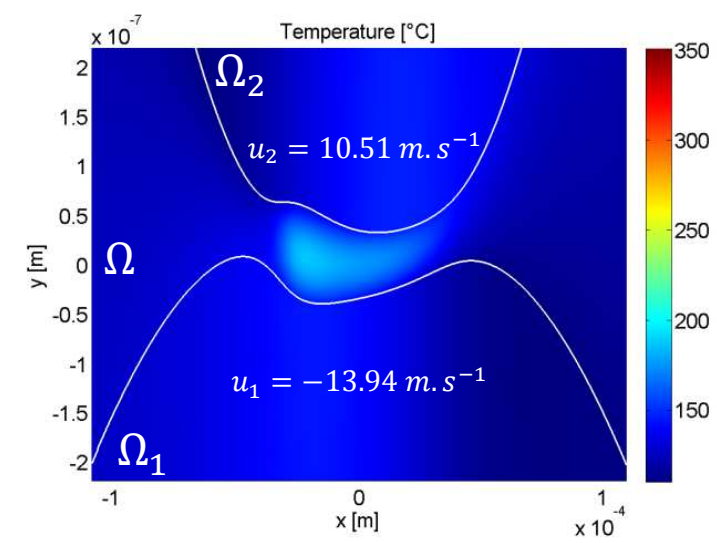

(f)

Fig. 7 Temperature profile in the lubricant $(\Omega)$ and in the cam $\left(\Omega_{2}\right)$ and follower $\left(\Omega_{1}\right)$ around the first reversal of entrainment obtained with the reference simulation. From (a) to (f), images correspond to camshaft angles $\theta$ of $117^{\circ}, 120^{\circ}, 123^{\circ}, 126^{\circ}, 129^{\circ}$ and $132^{\circ}$. 


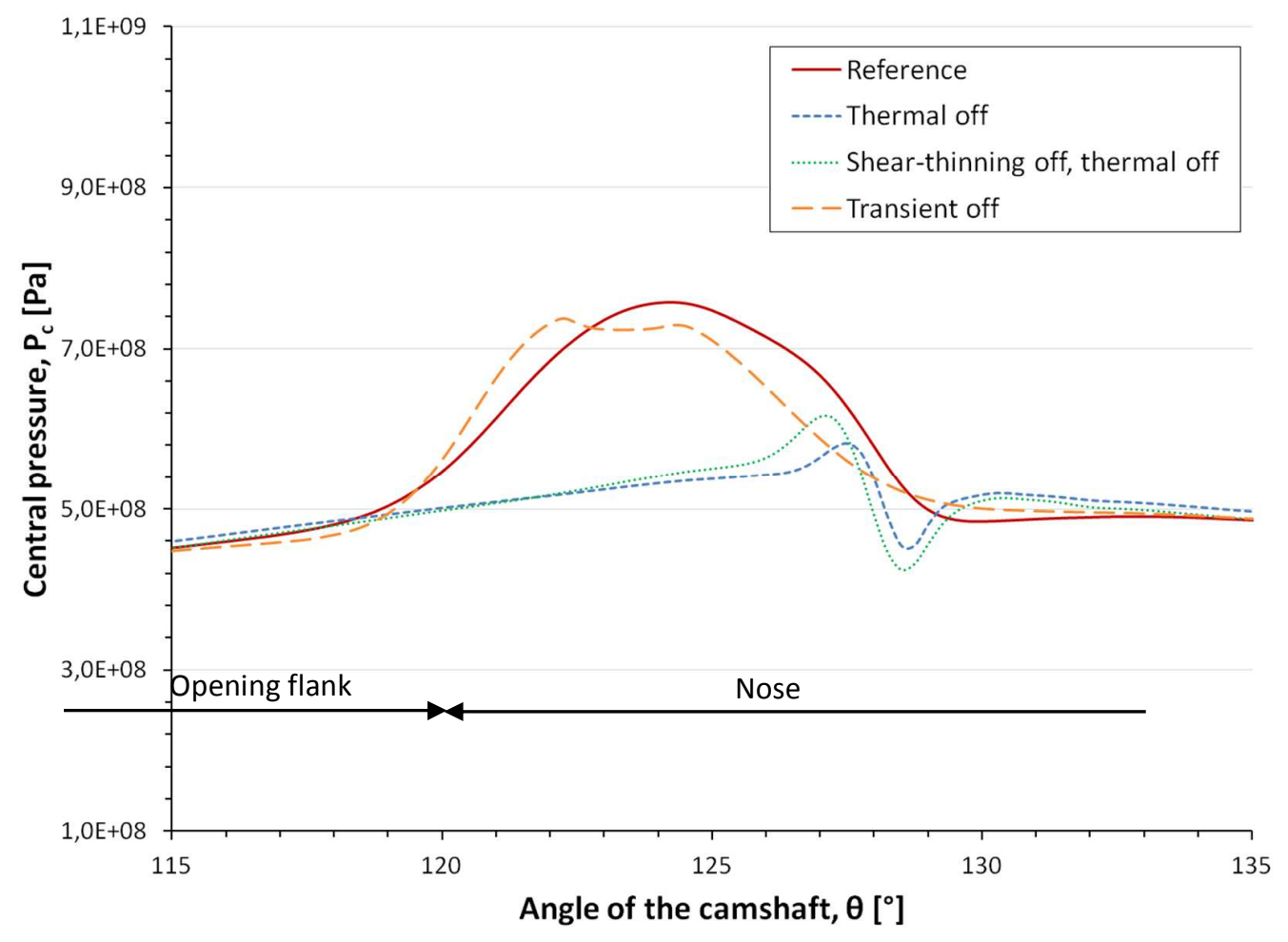

Fig. 8 Variation of the central pressure in the cam-follower contact around the first reversal of entrainment. 